\title{
The substrate-binding site in Cu nitrite reductase and its similarity to Zn carbonic anhydrase
}

Richard W. Strange1, Fraser E. Dodd'1,2, Zelda H.L. Abraham³, Gunter Grossmann', Thomas Bruser $^{3}$, Robert R.Eady ${ }^{3}$, Barry E. SMith ${ }^{3}$ and Samar Hasnain ${ }^{1}$

Nature Structural Biology 2, 287-292 (1995).

The following omissions were inadvertently made in our paper and are corrected below:

\begin{abstract}
Table 1 The explanation of superscript ' $b$ ' was omitted. This should have read:
' Godden et al. Science 253, 438-442 (1991). the Cu-ligand distances are identical for each monomer and are taken from unrefined coordinates of a monomer provided by Dr. E. Adman in November 1991. These distances are the same as given in the Brookhaven Protein Data Bank (INRD). The NiR trimer was generated by crystallographic symmetry. Figures 2 and 3 are also based on these coordinates.

Dr Adman has also recently brought to our attention a preliminary report indicating the structural similiarity of ACNiR and ACT2DNiR (Adman, E.T. \& Turley, S. (1993) Acta Crystallgr. A49 Suppl p. 75) and that the superposition of Asp 98 and His 255 with copper ligand residues of ascorbate oxidase was published in a preliminary form in reference 35 in our paper. The coordinates of our proposed model of nitrite bound $\mathrm{Cu}$ are available on request.
\end{abstract}

\section{Novel metal-binding proteins by design}

Michael Klemba', Kevin H. Gardner ${ }^{1}$, Stephen Marino', Neil D. Clarke² and Lynne Regan'1 Nature Structural Biology, 2 368-373 (1995).

The symbols for the $Z \beta 1$ variants $Z \beta 1 M$ and $Z \beta 1 L$ in the legends for Figures 3 and 4 were inadvertently switched. The corrected figure legends are printed below:

Fig. 3 Structural changes of the $Z \beta 1$ variants in reponse to metal. a, CD spectra of the apo form of $Z \beta 1 Z$ (circles), $Z \beta 1 M$ (squares) and $Z \beta 1 \mathrm{~L}$ (triangles) acquired at $2^{\circ} \mathrm{C} . b, C D$ spectra of the proteins at $2^{\circ} \mathrm{C}$ in the presence of 2.5 equivalents of $C \mathrm{dCl}, Z \beta 1 \mathrm{~A}$ (circles), $A \beta 1 M$ (squares) and $Z \beta 1 L$ (triangles). $Z n(i i)$ and $C d(i i)$ bound forms of the proteins gave similar spectra.

Fig. 4 The effect of metal binding on the stability of the Zb1 variants. $a$, Thermal denaturation of the apo form of $Z \beta 1 A$ (circles), $\mathrm{Z} \beta 1 \mathrm{M}$ (squares) and Z $\beta 1 \mathrm{~L}$ (triangles). Ellipticity at $220 \mathrm{~nm}$ is plotted as a function of temperture. The raw ellipticity data is shown, because $Z \beta 1 A$ and $Z \beta 1 M$ are not fully folded in the absence of metal, even at $2^{\circ} C$. $b$, Thermal denaturation of the $Z \beta 1$ variants in the presence of 1.1 molar equivalents of $C d C 12: Z \beta 1 A$ (circles), Z $\beta 1 M$ (squares) and Z $\beta 1 L$ (triangles). The fraction of unfolded protein is plotted as a function of temperature.

\section{The structure of calcyclin reveals a novel homodimeric fold for 5100 Ca2+-binding proteins}

Barbara C.M. Potts', Jarrod Smith1, Mikael Akke1,2, Thomas J. Macke1, Katsuo Okazaki², Hiroyoshi Hidaka ${ }^{3}$, David A.Case ${ }^{1}$ and Walter J. Chazin'

Nature Structural Biology 2, 790-796.

The following statement was inadvertently left out of the Acknowledgements: "supported in part by the Mondbusho International Scientific Research Program." 